\title{
Vascular leakage in chick embryos after expression of a secreted binding protein for fibroblast growth factors
}

\author{
Kevin McDonnell*, Emma T Bowden*, Rafael Cabal-Manzano, Becky Hoxter, \\ Anna T Riegel and Anton Wellstein \\ Lombardi Cancer Center, Georgetown University, Washington, DC, USA
}

\begin{abstract}
Fibroblast growth factors (FGFs) have been implicated in a variety of physiologic and pathologic processes from embryonic development to tumor growth and angiogenesis. FGFs are immobilized in the extracellular matrix of different tissues and require release from this storage site to trigger a response. Secreted FGF-binding proteins (FGF-BPs) can release immobilized FGFs, enhance the activity of locally stored FGFs and can thus serve as an angiogenic switch molecule in cancer. Here, we report on the effect of human FGF-BP transgene expression in chicken embryos. To establish the transgenic model, plasmid-based reporter vectors expressing luciferase, $\beta$-galactosidase or green fluorescent protein were introduced through different routes into 4- to 5day-old embryos grown outside their egg shell on top of the yolk sac. This allows for easy manipulation and continuous observation of phenotypic effects. Expression of human FGF-BP induced dose-dependent vascular permeability, hemorrhage and embryonic lethality. Light and electron microscopic studies indicate that this hemorrhage results from compromised microvascular structure. An FGF-1 expression vector with an added secretory signal mimicked this vascular leakiness phenotype whereas wild-type FGF-1 required coexpression of a threshold amount of FGF-BP. This model is a powerful tool for real-time monitoring of the effects of transient transgene expression during embryogenesis.
\end{abstract}

Laboratory Investigation (2005) 85, 747-755, advance online publication, 4 April 2005; doi:10.1038/labinvest.3700269

Keywords: chicken embryo; FGF; FGF-binding protein; transgene; vascular leakage

Fibroblast growth factors (FGFs) represent a large family of polypeptides that have been implicated in a variety of physiologic and pathologic processes including embryonic development, central nervous system growth, maintainance and repair as well as physiologic and tumor-associated angiogenesis and mitogenesis. ${ }^{1,2}$ FGF-1 and -2 and other less-abundant members of this growth factor family are typically stored in the extracellular matrix (ECM) bound to heparan sulfate proteoglycans (HSPG) and need to be released from this storage to trigger a biologic response. ${ }^{3,4}$ In addition, heparan sulfates on the surface of target cells are required by FGFs to trigger receptor oligomerization ${ }^{5-9}$ (reviewed in Pellegrini ${ }^{10}$ ). Two distinct mechanisms have been described by which locally stored FGFs can be

Correspondence: Dr A Wellstein, MD, PhD, Department of Oncology, Georgetown University, 3970 Reservoir Road, NW, Washington, DC 20057, USA.

E-mail: wellstea@georgetown.edu

*These two authors contributed equally to the work.

Received 22 March 2004; revised 6 February 2005; accepted 7 February 2005; published online 4 April 2005 delivered to their receptor: one mechanism involves digestion of the sugar backbone of HSPGs by heparinases or other glycosaminoglycan-degrading enzymes. ${ }^{11,12}$ Another mechanism is the binding of immobilized FGFs to secreted FGF-binding proteins (FGF-BPs) that serve as extracellular chaperones for FGFs. Wu et $a l^{13}$ originally isolated a human FGF-BP from the supernatants of A431 epidermoid carcinoma cells and it was found that mammalian FGF-BP can bind to FGF-1 and FGF-2 in a reversible manner, protect FGFs from degradation and present FGF-1 and -2 to high-affinity cell-surface receptors in an active form. ${ }^{13-18}$ Interestingly, heparin, heparansulfate and other heparinoids compete with FGF-BP binding to FGF-2 ${ }^{17,19}$ and more recently it was shown that FGF-BP interacts with perlecan, a heparansulfate proteoglycan present in the basement membrane. ${ }^{20}$ This demonstrates a direct interaction of the FGF-BP chaperone with cell matrix storage molecules for FGFs.

High levels of FGF-BP expression were observed in developing mouse embryo skin, gut and thymus during the second half of gestation. ${ }^{16,21}$ and expression was found downregulated after the neonatal 
period. Consistent with a role in cancer, FGF-BP expression was found to be induced early during malignant transformation of mouse and human skin $^{16,22}$ and colon epithelia, ${ }^{23}$ as well as upregulated in squamous cell carcinomas and colon cancers relative to normal tissues. ${ }^{14,15,20,23}$ Transient upregulation of FGF-BP was found during wound healing of mouse and human skin. ${ }^{22}$ On the other hand, retinoids were found to rapidly reduce expression of FGF-BP by transcriptional repression and reduction of mRNA stability in cultured tumor cells ${ }^{24,25}$ and in normal tissues and xenograft tumors in intact animals. ${ }^{26,27}$ Recombinant human FGF-BP enhances FGF-dependent angiogenesis, ${ }^{17}$ expression of FGF-BP in nontumorigenic cells can induce the formation of angiogenic tumors ${ }^{14}$ and depletion of FGF-BP from human colon cancer or squamous cell carcinoma cells by ribozyme-targeting showed a rate-limiting role for tumor growth and angiogenesis. ${ }^{15}$ Based on these studies in cancer, we proposed that upregulation of FGF-BP is one of the 'angiogenic switch' mechanisms required as essential steps in malignant progression. ${ }^{15,28}$

Here we sought to develop an animal model that allows transgene expression and continuous monitoring of potential phenotypic alterations. For this, chicken embryos were grown outside their shells in Petri dishes and we describe here a highly efficient plasmid-based gene transfer by injection of liposome-encapsulated expression vectors into the allantoic sac. Using this model, we report on a vascular leakage phenotype due to FGF-BP expression and subsequent embryonic lethality. We demonstrate that this phenotype is also induced by the expression of FGF-1 with an added signal peptide and that wild-type FGF-1 requires the coexpression of a threshold amount of FGF-BP to induce the phenotype.

\section{Materials and methods}

\section{In Vitro Cultivation of Embryos}

Three-day-old fertilized chicken (Gallus gallus) eggs (CBT Farms; Chestertown, MD, USA) were opened and intact embryos with yolk were placed in $10 \times 2 \mathrm{~cm}$ polystyrene culture dishes (Corning; Corning, New York, USA). Embryos were maintained in a humidified water-jacketed incubator at $39^{\circ} \mathrm{C}$ prior to and following transfection.

\section{Expression Vectors}

The human FGF-BP (hFGF-BP), green fluorescent protein (GFP), luciferase and $\beta$-galactosidase expression vectors were constructed employing the pRc/ CMV vector (Invitrogen, San Diego, CA, USA). The hFGF-BP plasmid contained the hFGF-BP open reading frame with a $\mathrm{C}$-terminal myc-epitope tag described previously. ${ }^{14,17}$ Expression vectors for a secreted FGF-1 and wild-type FGF-1 were kindly provided by Dr Stephen Zhan (American Red Cross, Rockville, MD, USA) and have been described elsewhere. ${ }^{29}$ Various concentrations of plasmid constructs were incubated with $25 \mu \mathrm{l}$ Lipofectamine ${ }^{\mathrm{TM}}$ reagent (Life Technologies) at room temperature for $45 \mathrm{~min}$. A measure of $25 \mu \mathrm{l}$ of the liposomal mixture were injected into the allantoic sac of the embryos using a Hamilton syringe (Hamilton; Reno, NV, USA) under $\times 3$ magnification with an Olympus SZH10 steromicroscope (see Figure 1a and b).

To quantitate gene transfer, CMV luciferase was used as a vector and embryos were harvested and homogenized in $150 \mu$ l Passive Lysis Buffer (Promega) at various time points following transfection. The Luciferase Reporter assay system (Promega) was used to detect firefly luciferase activity in $100 \mu \mathrm{l}$ homogenate utilizing a Monolight 2010 luminometer. Expressed FGF-BP was detected in paraffin-embedded sections from differently treated embryos. As a primary antibody, a commercially available anti-myc monoclonal antibody (Sigma) at 1:2000 was used to detect the myc tag in the exogenously expressed FGF-BP. The detection system of the antibody was as described for endogenous human FGF-BP. ${ }^{23}$
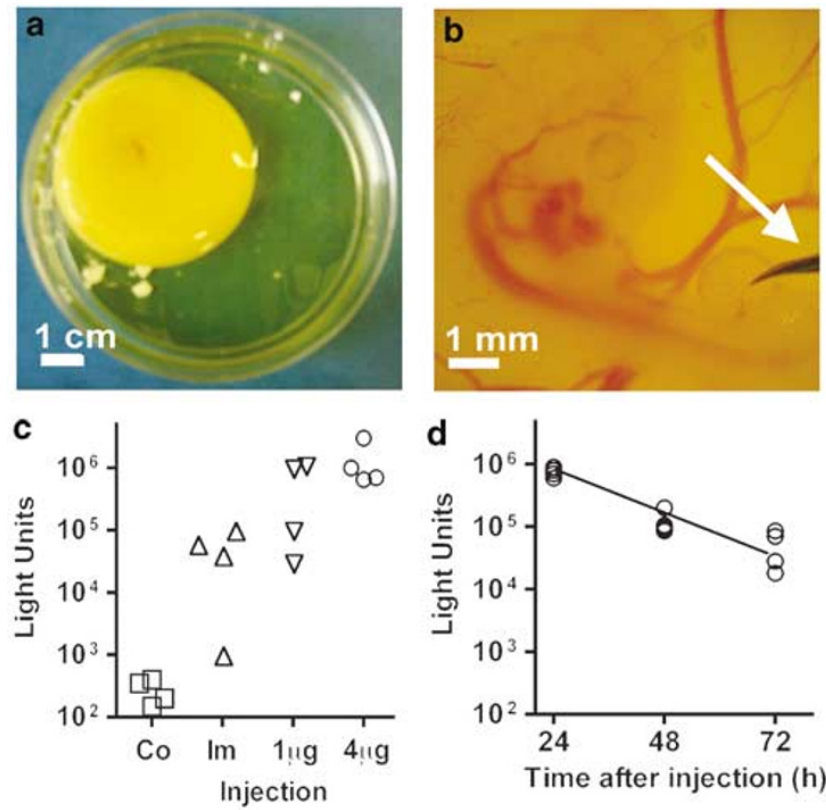

Figure 1 Gene expression in the chicken embryo. (a, b) Overview of the model, day 4 chicken embryos used for microinjection. The arrow in panel $\mathbf{b}$ points to the injection needle (black) placed into the allantoic sac. (c) Embryos were microinjected with $25 \mu \mathrm{l}$ of lipofectamine without (control) or with $1 \mu \mathrm{g}$ of CMV luciferase plasmid around the body of the embryo ( $\mathrm{Im}=\mathrm{DNA}$ immersion) or with 1 or $4 \mu \mathrm{g}$ of the plasmid into the allantoic sac. Luciferase activity was measured $24 \mathrm{~h}$ after microinjection. (d) Time course of luciferase activity after $4 \mu \mathrm{g}$ of CMV luciferase injection into the allantoic sac. Each data point represents measurements from an individual embryo. Note the logarithmic scale of the $Y$-axis. 


\section{Determination of Blood Vessel Integrity}

Embryonic blood vessel integrity was assessed by injection of $30 \mu \mathrm{l}$ Evan's Blue dye (Sigma, St Louis, MO, USA) (30 mg/kg in normal saline) into a chorioallantoic vein. Dye was allowed to circulate for $5 \mathrm{~min}$.

\section{Microscopy}

A Zeiss 410 confocal microscope at a resolution of 0.1323 pixels per micron using a $63 \times 11.4$ Zeiss immersion lens was used to visualize GFP expression in whole embryo specimens. Samples for light microscopic study were fixed in $10 \%$ formaldehyde/ PBS and then dehydrated using increasing concentrations of ethanol. After paraffin embedding, $10 \mu \mathrm{m}$ sections were obtained, xylene treated, rehydrated and then stained with hematoxylin and eosin (H\&E). Slides with tissue samples embedded in paraffin were incubated overnight at $55^{\circ} \mathrm{C}$, microwaved at high power for $10 \mathrm{~min}$, cooled for $5 \mathrm{~min}$ at room temperature and treated with xylene for $10 \mathrm{~min}$. After two washes in $100 \%$ ethanol for $5 \mathrm{~min}$, each of the slides was rehydrated and washed in $\mathrm{H}_{2} \mathrm{O}$ and phosphate-buffered saline (PBS) for $1 \mathrm{~min}$ each. Slides were then treated in PBS/1\% (vol/vol) citric acid at $80^{\circ} \mathrm{C}$ for $10 \mathrm{~min}$. The slides were then cooled to room temperature and washed twice in PBS. The tissue was blocked with 5\% goat normal serum in $\mathrm{PBS} / 2 \%$ bovine serum albumin for $30 \mathrm{~min}$. For immunohistochemistry, a polyclonal antibody to $\beta$ galactosidase was used. The antibody was applied overnight at $4^{\circ} \mathrm{C}$ and thereafter slides were washed three times in PBS/Tween. A biotinylated goat antirabbit secondary antibody (1:500) was then applied for $30 \mathrm{~min}$ at room temperature, followed by three washes in PBS/Tween. For detection and visualization, the ABC system was used according to the manufacturer's protocol with $0.1 \mathrm{mg} / \mathrm{ml}$ diaminobenzideine as substrate. Slides were counterstained with hematoxylin for $1 \mathrm{~min}$, dehydrated in ethanol and xylene, and coverslipped using a nonaqueous mounting medium.

Tissue sections for electron microscopic examination were glutaraldehyde fixed in phospho-buffered saline for $3 \mathrm{~h}$. Osmium tetroxide (1\%) fixation was then carried out for $1 \mathrm{~h}$ after which ultrathin sections were prepared. Uranyl acetate and lead citrate staining was performed and electron microscopic images generated employing a JEOL 1200 ex-transmission electron microscope.

\section{Statistical Evaluation}

The Prism 4 (Graphpad Inc.) program was used for data presentation, statistical analysis including the Kaplan-Meier survival analysis. $P$-values of $<0.05$ were considered statistically significant.

\section{Results}

\section{Gene Transfer in the Developing Chicken Embryo}

Different gene transfer methods were tested in embryos grown in Petri dishes outside their shell (Figure 1). A CMV-driven luciferase expression vector was microinjected along the dorsum of the embryo and allowed to spread over the entire embryo ('DNA immersion'; Figure 1c) or into the allantoic sac (see tip of the needle in Figure 1b). After $24 \mathrm{~h}$, the embryos were harvested and luciferase activity was measured in the whole embryos. Significant gene transfer and expression was achieved with DNA immersion and with allantoic injection. Allantoic sac injection, however, resulted in the highest levels of luciferase expression (Figure 1c).

Effective liposomal-mediated gene transfer depends on an optimal DNA to liposome ratio. To optimize the amount of gene transfer and expression, we assessed the effectiveness of different combinations. We observed that maximal gene transfer and expression was achieved using $4 \mu \mathrm{g}$ plasmid DNA together with $25 \mu$ l of lipofectamine (circles in Figure 1c). Increasing the amount of DNA beyond $4 \mu \mathrm{g}$ did not augment gene expression (data not shown).

\section{Time Course of Gene Expression}

Experimentally useful transgenesis requires that the transgene expression persists over a sufficient period of time. Embryos harvested at different times after gene transfer showed a maximal gene expression level around $24 \mathrm{~h}$ after dosing and we observed an exponential decline in expression levels with a half-life of $10 \mathrm{~h}$ using the CMV luciferase vector (Figure 1d). At $72 \mathrm{~h}$ postinjection, expression levels were still 100-fold above background suggesting that significant transgene expression can be achieved for several days.

\section{Spatial Pattern of Transgene Expression}

To assess the spatial pattern of transgene expression in the chick embryos, we used GFP as well as $\beta$ galactosidase as indicator molecules. Initially, $4 \mu \mathrm{g}$ of a CMV-driven GFP expression vector in $25 \mu \mathrm{l}$ of lipofectamine was microinjected into the allantois and $24 \mathrm{~h}$ later embryos were harvested and observed by confocal microscopy. GFP expression was evident both in the allantoic sac and diffusely throughout the entire embryo (Figure 2h). Histologic analysis was carried out using a parallel experimental series with a CMV-driven $\beta$-galactosidase expression vector. Low-power analysis revealed a striking $\beta$-galactosidase expression in the dorsal and ventral surface layers of the embryo as well as in the rostral region (Figure 2b). This expression was 

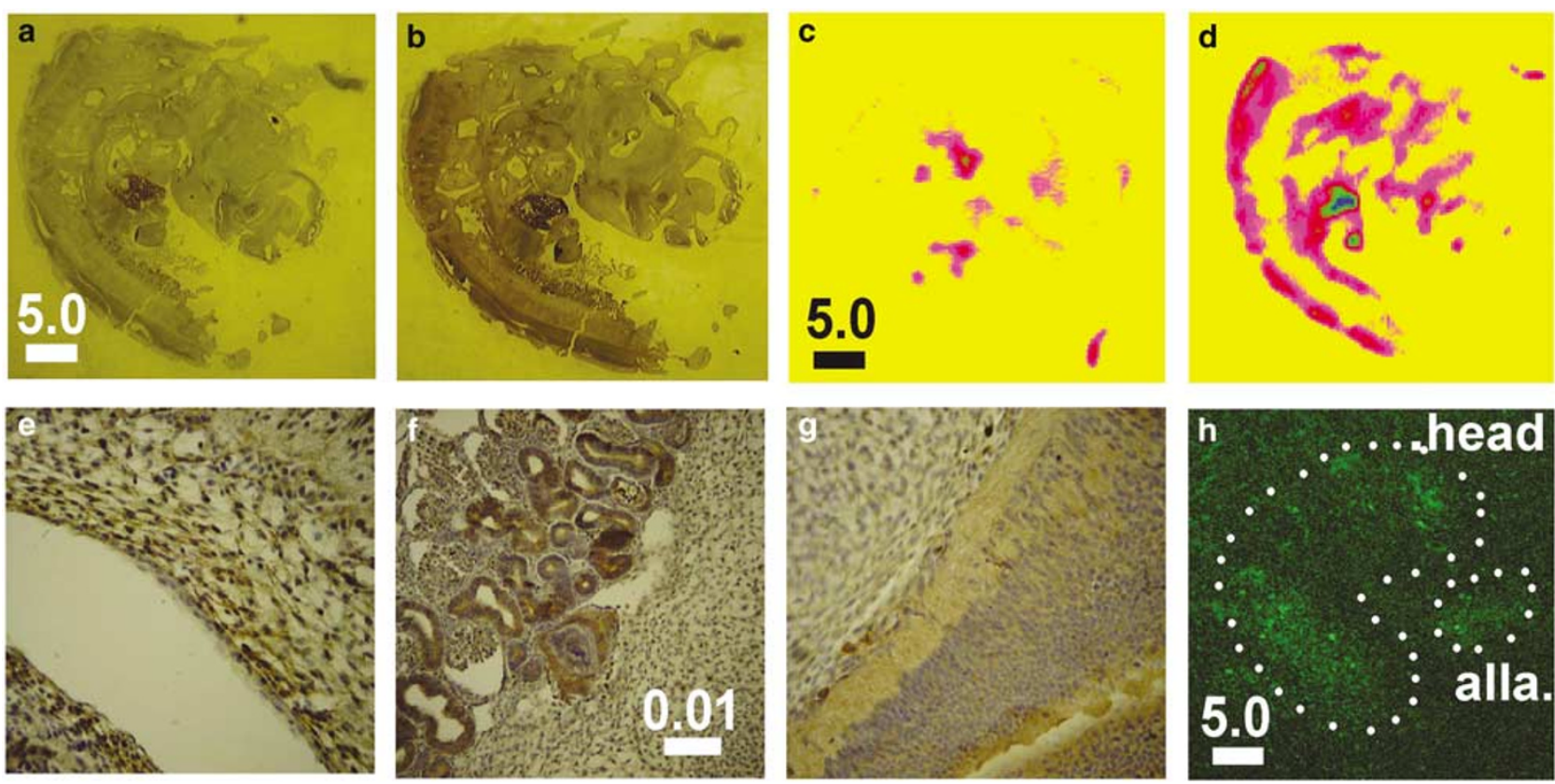

Figure 2 Spatial pattern of transgene expression in the chicken embryo. (a to $\mathbf{d}$ ) $\beta$-galactosidase as a reporter. In all, $4 \mu \mathrm{g}$ of the $\beta$ galactosidase expression vector or a control vector in $25 \mu \mathrm{l}$ lipofectamine were injected and embryos were killed and stained $24 \mathrm{~h}$ after injection. Control (a, c) and $\beta$-galactosidase (b, $\mathbf{d}$ to $\mathbf{g}$ ) injected embryos are shown. Pseudocolor images from control (c) and $\beta$ galactosidase injected embryo (d) show widespread expression of the reporter protein. Higher magnification images of the head region show expression around a blood vessel (e), in the kidney (f) and the neuroectodermal somites (g). (h) GFP as a reporter. In all, $4 \mu \mathrm{g}$ of the GFP expression vector in $25 \mu \mathrm{l}$ of lipofectamine was microinjected and embryos were examined by confocal microscopy to assess GFP expression $24 \mathrm{~h}$ later. Widespread expression in the whole embryo and the site of injection in the allantoic sac is seen. Size bars: mm.

absent in the control sections (Figure 2a). False color-based densitometry analysis of these sections revealed the regions of $\beta$-galactosidase expression more clearly (Figure 2c, d). Higher-power examination shows expression of $\beta$-galactosidase in vascular (Figure 2e), primitive kidney (Figure 2f) and neuroectodermal (Figure 2g) tissues. From this we conclude that the allantoic sac injection of a expression plasmid leads to a highly efficient gene transfer to a broad range of tissues in the embryo.

\section{Lethal Effect of FGF-BP In Vivo Due to Vascular Leakage}

We then used this chicken embryo transgenic model to monitor the impact of FGF-BP transgene expression. A CMV-driven expression vector was used to express the human FGF-BP. Luciferase inserted into the same vector or an empty expression vector without insert was used as a negative control. The human FGF-BP protein was expressed at high levels within $24 \mathrm{~h}$ after injection of the expression vector. Staining for the exogenously expressed FGF-BP protein using a monoclonal antibody that recognizes the C-terminal epitope tag in the FGF-BP transgene shows strong staining in different organs. Two examples of the brown appearing staining for FGF$\mathrm{BP}$ in tissues harvested $24 \mathrm{~h}$ after plasmid injection are depicted in Figure 3a, that is, the kidney and the retinal epithelia (Figure 3a, inset). Quite strikingly, the FGF-BP transgenic embryos exhibited a lower survival rate compared to the controls. Both microscopic (arrows in Figure 3b) and macroscopic (Figure 3c) hemorrhage into the extravascular space was observed in the embryos expressing FGF-BP. Also, the FGF-BP transgenic embryos exhibited mottled, and erythematous skin (Figure 3c). The induction of vascular leakage and subsequent embryo death due to FGF-BP expression was dosedependent (Figure 3d). At the lowest dose of FGFBP expression vector used ( $0.5 \mu \mathrm{g})$, embryo survival was not affected significantly relative to the control vector (used at $4 \mu \mathrm{g}$ ) but a significant effect was observed with $1 \mu \mathrm{g}$ as well as a further increase at $4 \mu \mathrm{g}$ of FGF-BP (Figure 3d).

To test the impact of FGF-BP expression on vascular integrity more directly, 4-day-old chicken embryos received FGF-BP or control plasmid, followed $48 \mathrm{~h}$ later by intravascular administration of Evans blue dye. The embryos were then evaluated for evidence of extravasation of the dye from the blood vessels. Macroscopically it was evident that the FGF-BP transgenic embryos exhibited increased extravasation of the dye as compared with the controls. (Figure 4a). Light microscopic ultrathin sections highlighted structural differences in blood vessels comparing the FGF-BP transgenic and control tissues. In the FGF-BP transgenic animals, the endothelial cells appeared stretched and two- to 
a

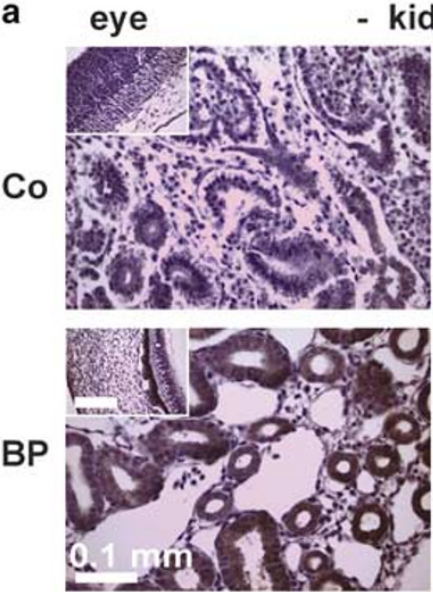

anti-BP

\section{c}
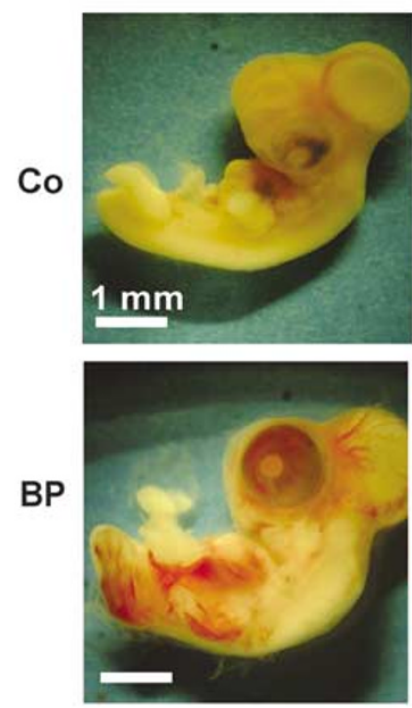

$+24 h$

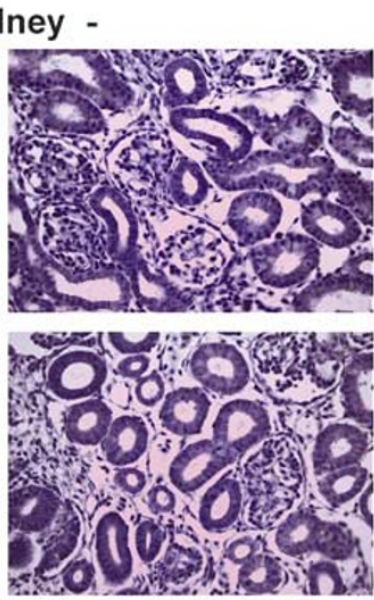

neg. control
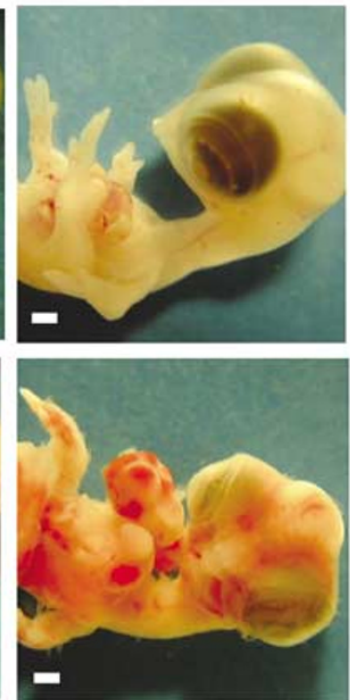

$+72 \mathrm{~h}$ b
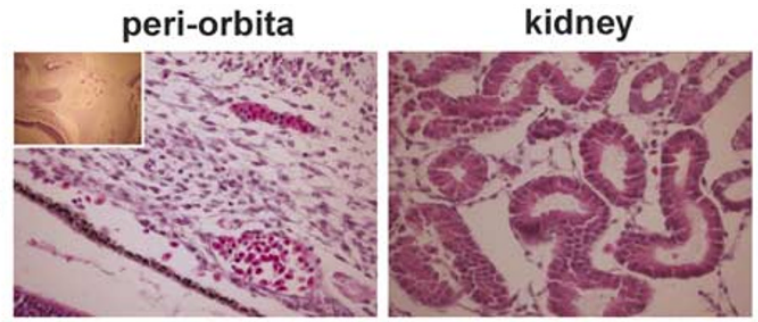

BP
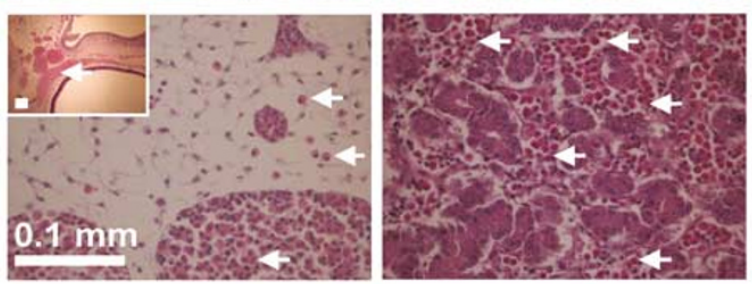

d

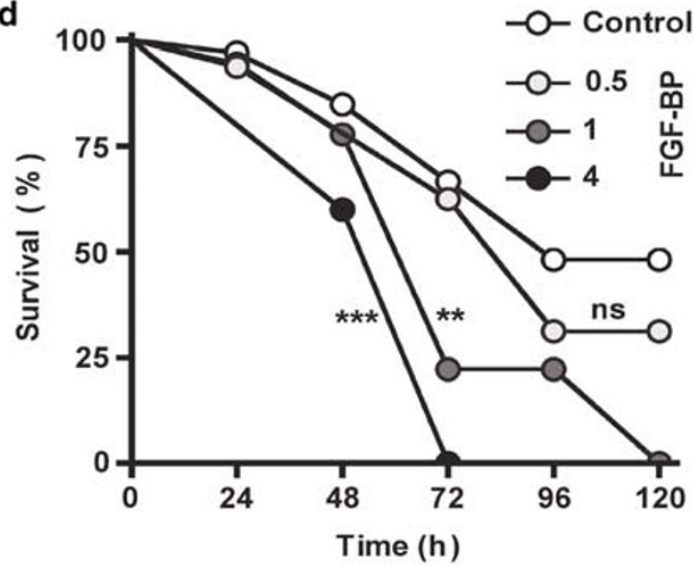

Figure 3 FGF-BP expression, vascular leakiness and embryonic survival. Day E4 embryos received microinjections of the FGF-BP (BP) or control (Co) expression vector into the allantoic sac. (a) Immunohistochemical detection of FGF-BP protein $24 \mathrm{~h}$ after injection of $4 \mu \mathrm{g}$ of the FGF-BP-expression vector. Staining of tissue sections with an anti-FGF-BP or control antibody shows expression of the protein (brown stain in the epithelia) in the animals receiving the FGF-BP expression vector. Kidney (major panel) and retina (inset) are shown as examples from control and FGF-BP-injected embryos. (b) H\&E staining showing extravasation of red blood cells (arrows) $72 \mathrm{~h}$ after injection. Periorbita (left; inset=overview) and kidney (right). (c) Macroscopic view of embryos harvested $24 \mathrm{~h}$ and $72 \mathrm{~h}$ after administration of the respective expression vectors. (d) Dose-response. Embryos received different doses of expression vector: FGF-BP $0.5 \mu \mathrm{g}(n=16), 1 \mu \mathrm{g}(n=18)$, or $4 \mu \mathrm{g}(n=15)$; control $4 \mu \mathrm{g}(n=33)$. Embryo survival is shown. ${ }^{* *} P<0.01 ;{ }^{* * *} P<0.001$; NS, $P>0.05$ relative to the control group.

three-fold fewer endothelial cells covered vessel segments in the FGF-BP group relative to the control (see Figure $4 \mathrm{~b}$ vs c). Also, erythrocytes were found in the tissues outside the vessel lumen in the FGF$\mathrm{BP}$ transgenics consistent with an initiation of vascular leakage at the capillary level already at this time point (arrows in Figure 4b; see also Figure $3 \mathrm{~b}$ for $72 \mathrm{~h}$ ). These features are in contrast to normal appearing endothelial cells and intact vasculature in the control tissue (Figure 4c). Electron microscopic analysis supported these findings and vessels from the FGF-BP transgenic showed ruffling and thinning of the cell membrane when compared to blood vessels from the controls (Figure 4d,e). Taken together, these microscopic data are consistent with an FGF-BP-induced disruption of blood vessel structure and integrity.

Based on these findings, we speculated that expression of a secreted member of the FGF family might mimic the effects observed with FGF-BP. Since FGF-BP is a secreted protein that is known to mobilize cell-bound FGFs, we hypothesized that secretion of an FGF might be required to induce the vascular leakiness phenotype observed here and 

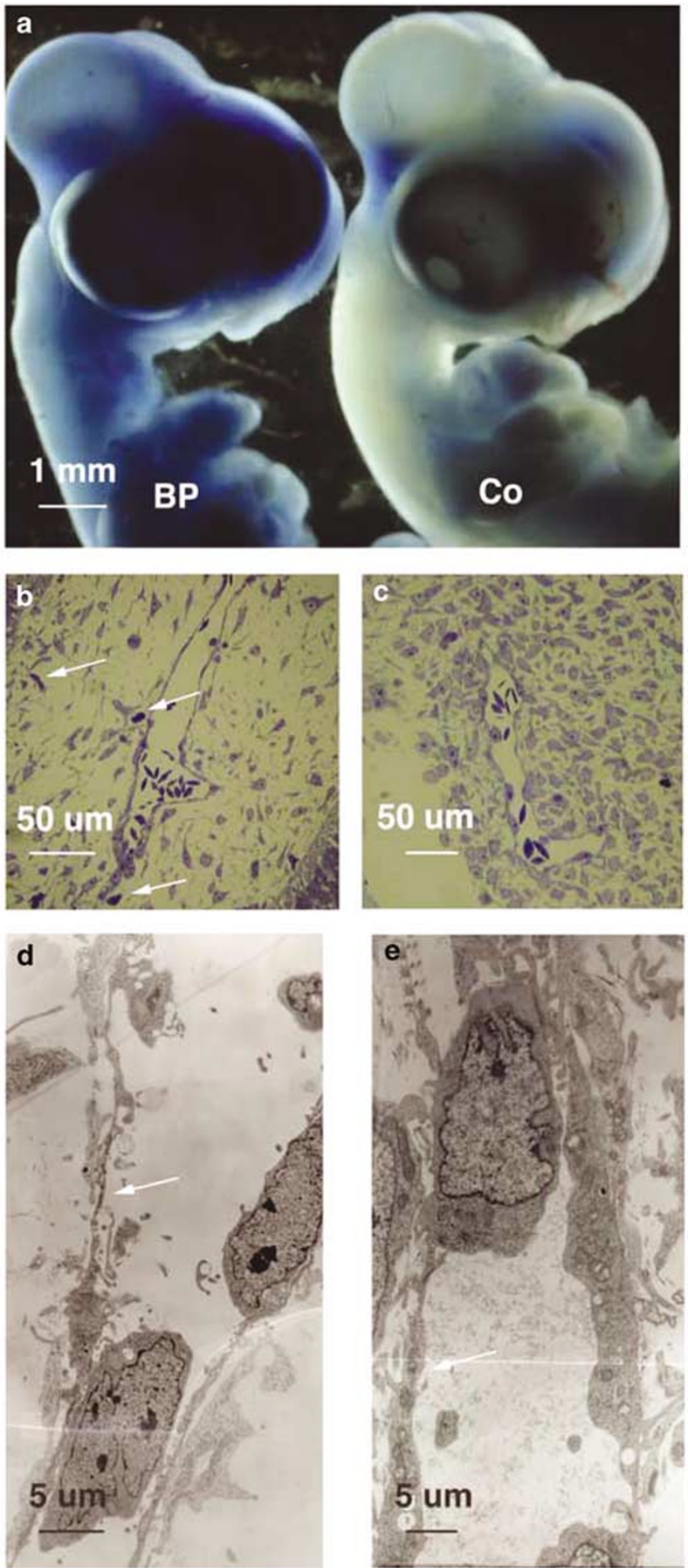

Figure 4 Effects of FGF-BP expression on vascular integrity. (a) Vascular retention of Evans blue dye $48 \mathrm{~h}$ after administration of FGF-BP or control vector. The pictures were taken 5 min after intravenous injection of the dye. (b,c) light microscopy of ultrathin sections. Stretched endothelial cells in the vasculature of tissues from an FGF-BP (b) relative to a control (c) transgenic embryo. Extravasated erythrocytes (arrows in panel b) indicate vascular leakage. (d,e) Electron microscopy. Attenuated cytoplasm and ruffling of the cell membranes (arrows) in the FGF-BP (d) relative to the control (e) embryo vasculature. opted for a direct comparison of a secreted vs nonsecreted (wild type) FGF-1. We obtained expression vectors for wild-type FGF-1 as well as an FGF-1 with a secretory signal peptide fused to the $\mathrm{N}$ terminus. These expression vectors for nonsecreted and secreted FGF-1 have been studied previously by different laboratories and shown to induce distinct phenotypes. ${ }^{29,30}$ Much to our surprise, expression of wild-type FGF-1, which remains cell bound and is not secreted, had no discernible effect on the embryos and showed no impact on their survival (Figure 5b). However, expression of FGF-1 containing a signal peptide induced vascular leakage and embryonic lethality indistinguishable from the phenotype observed after expression of FGF-BP (Figure 5a,b). As a final experiment, we then combined wild-type, nonsecreted FGF-1 with FGF$\mathrm{BP}$ to test whether they would cooperate in inducing the same phenotype. Indeed, the combination of FGF-1 and a low dose of FGF-BP, neither of which had a significant effect on their own, induced the same phenotype as the expression of secreted FGF-1. This supports the notion that FGF-BP can enhance the activity of FGF-1.

\section{Discussion}

Evaluation of gene function in an intact organism will generate significant new insight that cannot be gleaned from studies in cell culture. In parallel with the transient expression of genes in cultured cells, it is appealing to be able to apply the same vectors in a transient setting in vivo and avian embryos are a very appealing in vivo model system for this type of approach. A number of recent studies have reported liposomal-mediated gene transfer in the chick embryo kept in its shell involving 'in ovo' microinjection of early-stage embryos resulting in temporally and spatially significant transgenic expression. ${ }^{31-33}$ In the present study, we describe a novel approach that uses embryos growing on top of their yolk sacs in Petri dishes ('ex ovo') for continuous, real-time observation of phenotypic alterations and we demonstrate the efficiency of expression of different reporter genes after microinjection of lipidsome-encapsulated plasmid expression vectors. Injection into the allantoic sac proved an efficient route of transgene administration consistent with the known absorption of materials from the allantoic fluid. ${ }^{34-36}$ Different reporter genes showed that expression was widespread in somites, primitive epithelia, blood vessels and neuroectodermal tissues. Furthermore, gene expression persisted for at least $96 \mathrm{~h}$ following microinjection of the plasmid vectors. This model, thus allows for relatively easy, economical generation of a high number of transient transgenic animals, and permits direct and repeated observation of embryos during early stages of development and the impact of transgene expression. 
a
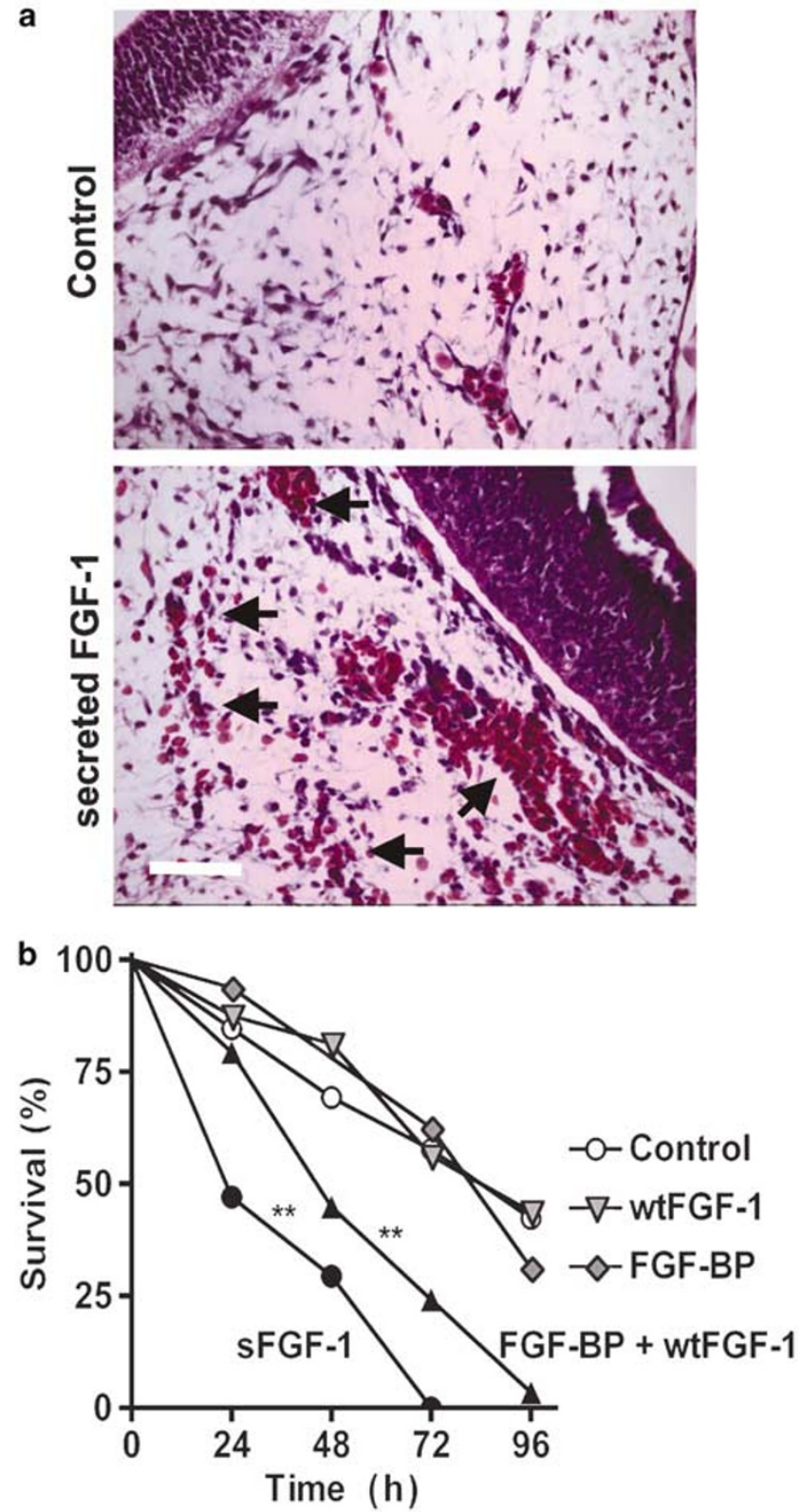

Figure 5 Effects of FGF-BP, wild-type FGF-1 and secreted FGF-1. (a) Effect of secreted FGF-1. Extravasation of red blood cells (arrows) into the periorbital space in embryos after expression of FGF-1 containing a secretory signal sequence. ${ }^{29}$ The size bar is $0.05 \mathrm{~mm}$. (b) Embryo survival after injection of different expression vectors: control $(n=26)$, wild-type FGF-1 (wtFGF-1, $n=16$ ), secreted FGF-1 (sFGF-1, $n=17)$, FGF-BP $(0.5 \mu \mathrm{g}, n=16)$ and FGFBP $(0.5 \mu \mathrm{g})$ plus wt FGF-1 $(n=29)$. No significant differences relative to control vector $(P>0.05)$ were found for wtFGF-1 and FGF-BP alone. ${ }^{*} P<0.01$ relative to control or FGF-BP alone or wtFGF-1 alone, respectively.

Previous work in our laboratory suggests that FGFBPs augment the effects of FGFs by solubilizing them from an extacellular sequestered state. ${ }^{15}$ In ongoing studies, we found that expression of FGF$\mathrm{BP}$ as a transgene in mice results in early embryonic lethality in the first half of gestation due to hemorrhage into the gestational sac (unpublished data). K14, MMTV or CMV-dependent transgene expression resulted in the same phenotype that was only distinguished by a slightly different onset between days E6 and E10 (unpublished data). The chicken embryo model reported here corroborates this effect and allowed for a more detailed analysis. Expression of FGF-BP in the chicken embryo produces dose-dependent effects that are evident as early as $24 \mathrm{~h}$ after gene transfer. Gross examination of the transgenic embryos revealed a mottling of the skin characterized by erythema and dilated, prominent vasculature consistent with hemorrhage. Intravascular injection of Evans blue dye with concomitant tissue extravasation among the FGFBP transgenic embryos confirmed this hemorrhagic phenotype. Light and electron microscopic photomicrographic examination of the transgenic embryonic tissue demonstrated FGF-BP-associated attenuation of endothelial cytoplasm together with a ruffled appearance of the cell membrane. Consistent with the vascular leakage, erythrocytes can also be detected extravascularly. Taken together, these data suggest a profound effect of FGF-BP on embryonic vascular development characterized by compromised vascular formation and/or maintenance.

It is likely that enhanced FGF activity due to FGF$\mathrm{BP}$ expression also increases the expression of Ang1, VPF/VEGF and/or their receptors as reported earlier by different laboratories. ${ }^{37-39}$ This imbalance of vascular growth and differentiation signals was reported to lead to disruption of vascular integrity and to produce a phenotype reminiscent of that observed in the current study. ${ }^{40,41}$ Our experiments comparing the effects of wild-type FGF-1 (without and with additional FGF-BP) and a form of FGF-1 engineered to be secreted supports the proposed mechanism of action of FGF-BP, that is, to enhance the activity of locally stored FGFs. Expression of wild-type FGF-1 alone did not impact on embryo viability, whereas inclusion of a secretory signal peptide with FGF-1 generated the same vascular leakage phenotype observed with FGF-BP. Interestingly, a combination of wild-type FGF-1 and a low dose of FGF-BP that had no effect on their own induced vascular leakage similar to the secreted FGF-1 and to FGF-BP at higher doses. This supports the notion of a positive interaction between the two molecules known from earlier work in other systems (see Introduction). It is tempting to speculate that differences observed with the secreted $v s$ the nonsecreted FGF-1 in some systems ${ }^{42}$ but not in others ${ }^{30}$ could be due to different levels of expression of an FGF chaperone molecule such as FGF-BP in either of these systems.

Finally, the induction of vascular permeability by very different mechanisms was shown to be a crucial step towards tumor angiogenesis. ${ }^{43-45}$ It is tempting to speculate that the effects of FGF-BP observed in the chicken embryo model reported here may be part of the function of FGF-BP as an angiogenic switch molecule in human cancers ${ }^{15,28}$ 
with vascular leakage resulting in activation of the local wound healing machinery.

\section{Acknowledgements}

We thank Dr Stephen Xie Zhan, American Red Cross, Rockville, MD for kindly providing the expression vectors for the wild-type and secreted FGF-1 as well as for helpful suggestions and discussion for the experiments. Drs Sandra McLeskey (University of Maryland, Baltimore) and Francis Kern (University of Alabama, Birmingham) provided conceptual help. We are grateful to Anne Dvorak and Harold Dvorak (Department of Pathology, Beth-Israel Hospital, Harvard Medical School) for their discussion on the interpretation of the microscopic analysis. This work was supported by the Grant NCI/NIH RO1 CA71508 to AW.

\section{References}

1 Mason IJ. The ins and outs of fibroblast growth factors. Cell 1994;78:547-552.

2 Powers CJ, McLeskey SW, Wellstein A. Fibroblast growth factors, their receptors and signaling. Endocr Relat Cancer 2000;7:165-197.

3 Kiefer MC, Stephans JC, Crawford K, et al. Ligandaffinity cloning and structure of a cell surface heparan sulfate proteoglycan that binds basic fibroblast growth factor. Proc Natl Acad Sci USA 1990;87:6985-6989.

4 Gould SE, Upholt WB, Kosher RA. Characterization of chicken syndecan-3 as a heparan sulfate proteoglycan and its expression during embryogenesis. Dev Biol 1995;168:438-451.

5 Yoneda A, Asada M, Oda Y, et al. Engineering of an FGF-proteoglycan fusion protein with heparin-independent, mitogenic activity. Nat Biotechnol 2000;18: 641-644.

6 Ballinger MD, Shyamala V, Forrest LD, et al. Semirational design of a potent, artificial agonist of fibroblast growth factor receptors. Nat Biotechnol 1999;17: 1199-1204.

7 Ogura K, Nagata K, Hatanaka $\mathrm{H}$, et al. Solution structure of human acidic fibroblast growth factor and interaction with heparin-derived hexasaccharide. J Biomol NMR 1999;13:11-24.

8 Plotnikov AN, Schlessinger J, Hubbard SR, et al. Structural basis for FGF receptor dimerization and activation. Cell 1999;98:641-650.

9 Schlessinger J, Plotnikov AN, Ibrahimi OA, et al. Crystal structure of a ternary FGF-FGFR-heparin complex reveals a dual role for heparin in FGFR binding and dimerization. Mol Cell 2000;6:743-750.

10 Pellegrini L. Role of heparan sulfate in fibroblast growth factor signalling: a structural view. Curr Opin Struct Biol 2001;11:629-634.

11 Bashkin P, Doctrow S, Klagsbrun M, et al. Basic fibroblast growth factor binds to subendothelial extracellular matrix and is released by heparitinase and heparin-like molecules. Biochemistry 1989;28: 1737-1743.
12 Vlodavsky I, Bashkin $\mathrm{P}$, Ishai-Michaeli $\mathrm{R}$, et al. Sequestration and release of basic fibroblast growth factor. Ann NY Acad Sci 1991;638:207-220.

$13 \mathrm{Wu}$ D, Kan M, Sato GH, et al. Characterization and molecular cloning of a putative binding protein for heparin-binding growth factors. J Biol Chem 1991;266: 16778-16785.

14 Czubayko F, Smith RV, Chung HC, et al. Tumor growth and angiogenesis induced by a secreted binding protein for fibroblast growth factors. J Biol Chem 1994;269:28243-28248.

15 Czubayko F, Liaudet-Coopman ED, Aigner A, et al. A secreted FGF-binding protein can serve as the angiogenic switch in human cancer [see editorial]. Nat Med 1997;3:1137-1140.

16 Kurtz A, Darwiche N, Harris V, et al. Expression of a binding protein for FGF is associated with epithelial development and skin carcinogenesis. Oncogene 1997;14:2671-2681.

17 Tassi E, Al-Attar A, Aigner A, et al. Enhancement of fibroblast growth factor (FGF) activity by an FGF-binding protein. J Biol Chem 2001;276:4024740253.

18 Lametsch R, Rasmussen JT, Johnsen LB, et al. Structural characterization of the fibroblast growth factorbinding protein purified from bovine prepartum mammary gland secretion. J Biol Chem 2000;275: 19469-19474.

19 Aigner A, Butscheid M, Kunkel P, et al. An FGFbinding protein (FGF-BP) exerts its biological function by parallel paracrine stimulation of tumor cell and endothelial cell proliferation through FGF-2 release. Int J Cancer 2001;92:510-517.

20 Mongiat M, Otto J, Oldershaw R, et al. Fibroblast growth factor-binding protein is a novel partner for perlecan protein core. J Biol Chem 2001;276: 10263-10271.

21 Aigner A, Ray PE, Czubayko F, et al. Immunolocalization of an FGF-binding protein reveals a widespread expression pattern during different stages of mouse embryo development. Histochem Cell Biol 2002;117: $1-11$.

22 Kurtz A, Aigner A, Cabal-Manzano R, et al. Differential regulation of an FGF-binding protein during skin carcinogenesis and wound healing. Neoplasia 2004, in press.

23 Ray R, Cabal-Manzano R, Moser AR, et al. Upregulation of fibroblast growth factor-binding protein, by beta-catenin during colon carcinogenesis. Cancer Res 2003;63:8085-8089.

24 Liaudet-Coopman EDE, Wellstein A. Regulation of gene expression of a binding protein for fibroblast growth factor by retinoic acid. J Biol Chem 1996;271: 21303-21308.

25 Boyle BJ, Harris VK, Liaudet-Coopman ED, et al. Differential regulation of a fibroblast growth fatorbinding protein by receptor-selective analogs of retinoic acid. Biochem Pharmacol 2000;60:16772684.

26 Aigner A, Malerczyk C, Houghtling R, et al. Tissue distribution and retinoid-mediated downregulation of an FGF- binding protein (FGF-BP) in the rat. Growth Factors 2000;18:51-62.

27 Liaudet-Coopman EDE, Berchem GJ, Wellstein A. In vivo inhibition of angiogenesis and induction of apoptosis by retinoic acid in squamous cell carcinoma. Clin Cancer Res 1997;3:179-184. 
28 Rak J, Kerbel RS. bFGF and tumor angiogenesis-back in the limelight? [news; comment]. Nat Med 1997;3: 1083-1084.

29 Forough R, Xi Z, MacPhee M, et al. Differential transforming abilities of non-secreted and secreted forms of human fibroblast growth factor-1. J Biol Chem 1993;268:2960-2968.

30 Jouanneau J, Gavrilovic J, Caruelle D, et al. Secreted or nonsecreted forms of acidic fibroblast growth factor produced by transformed epithelial cells influence cell morphology, motility, and invasive potential. Proc Natl Acad Sci USA 1991;88:2893-2897.

31 Muramatsu T, Mizutani Y, Ohmori Y, et al. Comparison of three nonviral transfection methods for foreign gene expression in early chicken embryos in ovo. Biochem Biophys Res Commun 1997;230:376-380.

32 Itasaki N, Bel-Vialar S, Krumlauf R. 'Shocking' developments in chick embryology: electroporation and in ovo gene expression. Nat Cell Biol 1999;1: E203-E207.

33 Pekarik V, Bourikas D, Miglino N, et al. Screening for gene function in chicken embryo using RNAi and electroporation. Nat Biotechnol 2003;21:93-96.

34 Busch Mt, Milakofsky L, Hare T, et al. Regulation of substances in allantoic and amniotic fluid of the chicken embryo. Comp Biochem Physiol A 1997;116: 131-136.

35 Stewart ME, Terepka AR. Normal histology and evidence for active electrolyte transport from the allantoic fluid. Exp Cell Res 1969;58:93-106.

$36 \mathrm{McNabb}$ RA. Urates and allantoic regulation in embryonic Japanese quail, corturnix cortunix japonica. J Exp Zoo 1986;240:9-15.

37 Hata Y, Rook SL, Aiello LP. Basic fibroblast growth factors induce expresson of VEGF receptor KDR through a protein kinase C and p44/p42 mitogenactivated protein kinase-dependent pathway. Diabetes 1999;48:1145-1155.

38 Mandriota SJ, Pepper MS. Regulation of angiopoietin-2 mRNA levels in bovine microvascular endothelial cells by cytokines and hypoxia. Circ Res 1998;83: 852-859.

39 Seghezzi G, Patel S, Ren CJ, et al. Fibroblast growth factor-2 (FGF-2) induces vascular endothelial growth factor (VEGF) expression in the endothelial cells of forming capillaries: an autocrine mechanism contributing to angiogenesis. J Cell Biol 1998;141: 1659-1673.

40 Larcher F, Murillas R, Bolontrade M, et al. VEGF/ $\mathrm{VPF}$ overexpression in skin of transgenic mice induces angiogenesis, vascular hyperpermeability and accelerated tumor development. Oncogene 1998; 17:303-311.

41 Maisonpierre PC, Suri C, Jones PF, et al. Angiopoietin2, a natural antagonist for Tie2 that disrupts in vivo angiogenesis. Science 1997;277:55-60.

42 Forough R, Xi Z, MacPhee $\mathrm{M}$, et al. Differential transforming abilities of non-secreted and secreted forms of human fibroblast growth factor-1. J Biol Chem 1993;268:2960-2968.

43 Senger DR, Van De Water L, Brown LF, et al. Vascular permeability factor (VPF, VEGF) in tumor biology. Cancer Metastasis Rev 1993;12:303-324.

44 Gratton JP, Lin MI, Yu J, et al. Selective inhibition of tumor microvascular permeability by cavtratin blocks tumor progression in mice. Cancer Cell 2003; 4:31-39.

45 Dvorak HF. Rous-Whipple Award Lecture. How tumors make bad blood vessels and stroma. Am J Pathol 2003;162:1747-1757. 